\title{
Effect of Ramadan Fasting on the Ability of Serum, PBMC and Macrophages from Healthy Subjects to Kill M. Tuberculosis
}

\author{
Ardik Lahdimawan ${ }^{1}$, Kusworini Handono ${ }^{2}$, M. Rasjad Indra ${ }^{3}$, \\ Sumarno Reto Prawiro ${ }^{4}$

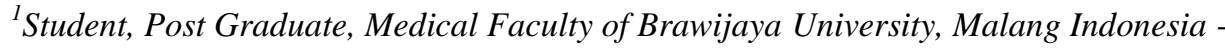 \\ Neurosurgery Department, Medical Faculty of Lambung Mangkurat University, Banjarmasin Indonesia. \\ ${ }^{2}$ Department of Clinical Pathology, Medical Faculty of Brawijaya University, Malang Indonesia. \\ ${ }^{3}$ Department of Physiology, Medical Faculty of Brawijaya University, Malang Indonesia. \\ ${ }^{4}$ Laboratory of Microbiology, Medical Faculty of Brawijaya University, Malang Indonesia.
}

\begin{abstract}
Objective: The objective of this study was to determine whether Ramadan fasting (RF) alters the ability of serum, peripheral blood mononuclear cells (PBMC) and macrophages to kill Mycobacterium tuberculosis (M. tb).

Methods: Thirty healthy male volunteers who fasted during Ramadan participated in the study. Blood sampling was conducted seven days before and on the seventh and $21^{\text {st }}$ day of Ramadan. The following parameters were measured by ELISA: Complement C3, inducible nitric oxide synthase (iNOS), superoxide dismutase (SOD) levels in serum and PBMC. The killing ability of immune components from these volunteers was determined by measuring colony-forming units (CFUs).

Results: The CFU in PBMC infected with M. tb (PIM) and macrophages infected with M. tb (MIM) decreased on the seventh day of RF compared with that observed before RF. The CFUs in serum infected with M. tb (SIM) increased and CFUs in MIM decreased on the $21^{\text {st }}$ day of RF compared with before RF. The levels of iNOS in serum and the CFUs in MIM decreased and the CFUs in SIM and PIM increased on the $21^{\text {st }}$ day of RF compared with the seventh day of RF. The levels of iNOS in PBMC significantly increased $(p<0.05)$ on the seventh and $21^{\text {st }}$ day of Ramadan compared with before $R F$. The other differences were not significantly different.

Conclusions: This study suggests that RF has a beneficial effect on host defence against $\mathrm{M}$. tb and decreases the risk of tuberculosis (TB) infection in healthy subjects.
\end{abstract}

Keywords: killing ability; Mycobacterium tuberculosis; Ramadan fasting.

\section{Introduction}

The Ramadan fasting (RF) period is associated with altered meal patterns, sleep and ritual habits in Muslims [1] that changes the body's circadian rhythm from diurnal to nocturnal and acts as a stressor to which the body will respond. The stress from RF induces different stress responses in serum, peripheral blood mononuclear cells (PBMC) and macrophages [2]. Stress may lead to decreased immune functions and thus to clinical disease [3]. Various types of physiological stressors, including physical exercise and emotional stress, i.e., RF, can strongly influence immune function [4,5]. Several studies conducted by Holmes have suggested that individuals who have experienced stressful situations, such as divorce, death of a spouse or loss of a job, were more likely to develop tuberculosis (TB) and less likely to recover from this disease [3].

In this study, we examined the effect of fasting during the lunar month of Ramadan on superoxide dismutase (SOD) and inducible nitric oxide synthase (iNOS) levels in serum and PBMC and the killing ability of serum, PBMC and macrophages from a group of 30 healthy subjects against $M$. $t b$. The main emphasis of this study was to determine the killing ability of serum, PBMC and macrophages during RF, as little is known about this topic.

Mycobacterium tuberculosis (M. $t b$ ) is the microorganism that is responsible for TB, a disease that annually affects 8 to 9 million people worldwide and accounts for approximately 2 to 3 million deaths each year $[6,7]$. The prevalence of TB started to increase in some developed countries in the mid-1980s for three principal reasons. First, there have been population increases in areas of the world where TB is the most prevalent, particularly in Central Africa and South Asia. Second, increases in TB have resulted from increases in human immunodeficiency virus (HIV). Finally, as more people worldwide live in poverty, where poor nutrition and crowded conditions can lead to the spread of TB, the disease risk will be compounded [8]. India, China, Indonesia, Bangladesh and Pakistan, the most heavily populated countries in Asia, have the highest incidence of TB and together, have over half of the cases in the world [6,7]. 
The natural history of TB shows that most individuals are resistant to the infection, most likely due to their capacity to generate an efficient immune response to $M$. $t b$. However, such individuals are incapable of completely eliminating the bacteria. Of the individuals exposed to $M$. $t b, 10$ to $30 \%$ become infected [6]. It is estimated that of 100 people newly infected with the tubercle bacilli, only approximately 5-10 individuals will develop TB over their lifetime [6,9,10]. Consequently, TB can be disseminated or localised in pulmonary, lymph node, renal, or bone forms and can also affect any other organ. [6,10].

In immunocompetent hosts, the innate and adaptive arms of the immune system are relatively efficient at containing and killing microbial pathogens [11]. After the barrier system's first line of defence, the second line consists of local factors and cells that are activated or recruited to the site of microbial invasion. These recruited components include the complement system and resident macrophages [12].

In the early phases of an infection, the complement cascade can be activated on the surface of a pathogen [12], and macrophages play an essential role in the immune response against $M$. $t b$. The immune response against $M . t b$ is complex. Macrophages play a unique role in the host response, as they are both the primary effector cells for bacterial killing and the primary habitat in which the persisting bacilli reside [13]. In healthy humans, PBMC are the only easily available immune cells [14]. Human monocytes ingest $M$. $t b$ by conventional phagocytosis, as viewed by electron microscopy [15]. Compared to monocytes, fewer $M$. $t b$ organisms were taken up by PBMC, and serum did not have a major effect on the uptake of $M . t b[16]$.

\section{Materials And Methods}

\section{Human Serum, PBMC and Macrophages}

Blood samples were drawn from all participants for examination of complement C3 levels and SOD and iNOS levels in serum and PBMC, and of the ability of serum, PBMC and macrophages to kill $M$. $t b$. Samples were drawn between 9 am and $12 \mathrm{pm}$ to avoid diurnal variation at 7 days before Ramadan and on the $7^{\text {th }}$ and $21^{\text {st }}$ day of RF [2].

Ten millilitres of venous blood from the antecubital vein was collected in an ethylenediaminetetraacetic acid (EDTA) vial and divided in two parts for the isolation of serum and cells. The plasma was separated and kept at $-80^{\circ} \mathrm{C}$ until analysis [2,17]. PBMC were isolated using dextran sedimentation and Hypaque-Ficoll density-gradient separation followed by hypotonic lysis of erythrocytes as previously described $[2,18]$. Human monocytes were isolated from buffy coats and cultured for 6 days in 24- or 6-well tissue culture plates (Corning, Corning, NY) as previously described [19] with a minor modification: cells were cultured in RPMI 1640 with $1 \%$ Pen/Strep (Sigma Aldrich), $0.2 \%$ serum-free NaBic (bioworld) and 10\% foetal bovine serum (FBS) (Gibco Inc.), $\mathrm{pH} 7.2$ and incubated at $37^{\circ} \mathrm{C}$ in the presence of $5 \% \mathrm{CO}_{2}$. Culture medium and nonadherent cells were removed by aspiration every 3 days, and monolayers were subsequently incubated with fresh culture medium supplemented with $10 \%$ autologous serum $[2,5,20]$.

\section{Microorganism}

The stock of $M . t b \mathrm{H} 37 \mathrm{Rv}$ was grown at $37^{\circ} \mathrm{C}$ in $100 \mathrm{ml}$ of Middlebrook $7 \mathrm{H} 9$ broth for 3 weeks [21]. Colonies were mixed with phosphate-buffered saline (PBS), vortexed with glass beads, and centrifuged at 6000 rpm for 15 minutes. The cell pellets were then washed twice with PBS and the optical density (OD) per $10^{-5} \mathrm{ml}$ was determined using a spectrophotometer at $620 \mathrm{~nm}$ and bacteria were diluted to $10^{4}$ bacteria/ml.

\section{$M . t b$ infections of human serum, PBMC and Macrophages}

Human serum $(100 \mu \mathrm{l})$, PBMC $\left(5 \times 10^{2} / \mathrm{ml}\right)$ and macrophages $\left(5 \times 10^{2} / \mathrm{ml}\right)$ each infected with $M$. $t b$ $\left(10^{4} / \mathrm{ml}\right)$ at a multiplicity of infection (MOI) of 20 . The microbes and human samples were then incubated for 3 hours in shaking water bath at $37^{\circ} \mathrm{C}$. PBMC and macrophages were washed 3 times to remove all extracellular bacilli. Intracellular bacteria were obtained by lysing the cells with sterile PBS containing $0.1 \%$ saponin (Sigma, St Louis, MO), and released bacilli were serially diluted in PBS containing 0.01\% Tween-80 (Merck, Darmstadt, Germany). Finally, bacteria were placed in culture in duplicate on Middlebrook 7H10 agar with oleic acid-albumin-dextrose-catalase (OADC) as previously described [13] with the slight modification of the addition of $\mathrm{CO}_{2}$ overnight. CFUs were counted after 14 days of incubation at $37^{\circ} \mathrm{C}$ and plates were maintained for 30 days to ensure that no additional CFUs appeared.

\section{ELISA}

Purified PBMC from each individual were resuspended in incubation buffer (sterile pyrogen-free Hanks balanced salt solution [HBSS; Bio-Whittaker]), vortexed and centrifuged at $1400 \mathrm{rpm}$ for 7 minutes at $4^{\circ} \mathrm{C}$. Pellets were then resuspended in RIPA buffer with PIC and PMSF and the solution was vortexed and incubated on ice for 30 minutes. Following, incubation, these samples were centrifuged at 12,000 rpm for 20 minutes at $4{ }^{\circ} \mathrm{C}$. The supernatant was then stored at $-20^{\circ} \mathrm{C}$ until analysis [2]. Complement C3, iNOS, and SOD 
levels in serum and PBMC were estimated in duplicate in an enzyme-linked immunosorbent assay (ELISA) using a commercial ELISA kit (R\&D Systems).

\section{Statistical Analysis}

To perform a statistical analysis of these data, means were calculated and one-sample KolmogorovSmirnov tests, ANOVAs and Duncan tests were performed using SPSS version 11.5 for Windows.

\section{Results}

The study population consisted of healthy males who were medical students at the medical school of Brawijaya University. Due to various limitations including the need for laboratory manipulation, fresh blood to be immediately examined, and adequate blood volumes, most blood samples were unsuitable for use in this study. In total, only 13 cases were eligible for the measurement of the SOD level in PBMC, 14 cases were eligible for the measurement of $\mathrm{C} 3$ and iNOS levels in serum and PBMC, 16 cases were eligible for the measurement of the killing ability of serum and PBMC, and 30 cases were eligible for measurement of the killing ability of macrophages. The subjects ranged in age from 18-22 years (mean \pm SD: $20.26 \pm 1.13$ years).

Our results reveal that on $7^{\text {th }}$ day and $21^{\text {st }}$ day of Ramadan, the iNOS levels of PBMCs were significantly increased $(\mathrm{p}<0.05)$ compared with the levels in PBMCs before RF. On $7^{\text {th }}$ day of Ramadan, the CFUs from PBMCs infected with $M$. $t b$ (PIM) and the CFUs from macrophages infected with $M . t b$ (MIM) decreased significantly $(\mathrm{p}<0.05)$ compared to the CFUs obtained from cells isolated before RF. On $21^{\text {st }}$ day of Ramadan, the CFUs from serum infected $M$. $t b$ (SIM) increased and the CFUs from MIM decreased significantly $(\mathrm{p}<0.05)$ compared to the CFUs from samples isolated before RF. When samples obtained on the $21^{\text {st }}$ day of Ramadan were compared to samples obtained on the $7^{\text {th }}$ day of Ramadan, the iNOS levels of PBMC were observed to decrease, the CFUs of SIM and PIM were shown to increase, and the CFUs of MIM decreased significantly $(\mathrm{p}<0.05)$. The other differences were not statistically significant (Table 1$)$.

Table 1. Mean C3, SOD and iNOS levels in serum and PBMC and CFUs of SIM, PIM and MIM before RF and on the $7^{\text {th }}$ and $21^{\text {st }}$ day of Ramadan. Duncan test 1) before Ramadan compared to $7^{\text {th }}$ day, 2) before Ramadan compared to $21^{\text {st }}$ day, 3) $7^{\text {th }}$ day compared to $21^{\text {st }}$ day. (* = Significant).

\begin{tabular}{lccc}
\hline & Before RF & $7^{\text {th }}$ day & $21^{\text {st }}$ day \\
\hline Serum C3 & $3606.8143 \pm 217.36752$ & $3657.0286 \pm 335.31663$ & $3504.1786 \pm 366.96462$ \\
Serum SOD & $84.1620 \pm 7.57224$ & $88.0060 \pm 7.58655$ & $88.1840 \pm 6.10583$ \\
Serum iNOS & $591.6000 \pm 124.02236$ & $646.1929 \pm 166.45693$ & $583.5357 \pm 147.10329$ \\
PBMC SOD & $81.5592 \pm 11.87552$ & $79.8054 \pm 10.65989$ & $77.9194 \pm 9.70652$ \\
PBMC iNOS & $514.9786 \pm 86.46724$ & $1376.2357 \pm 167.88722(*)$ & $979.0786 \pm 85.60523(*)$ \\
CFUs of SIM & $31.81 \pm 29.989$ & $26.56 \pm 21.416$ & $101.88 \pm 47.162(*)$ \\
CFUs of PIM & $41.38 \pm 24.077$ & $16.19 \pm 15.766\left(^{*}\right)$ & $51.94 \pm 30.267\left(^{*}\right)$ \\
CFUs of MIM & $92.43 \pm 33.508$ & $46.13 \pm 16.192\left(^{*}\right)$ & $3.73 \pm 2.434(*)$ \\
\hline
\end{tabular}

\section{Discussion}

The infectious cycle of $M$. $t b$, as it relates to the disease TB in humans, begins with the release of the bacilli in fine aerosol particles when an individual with active TB coughs or sneezes [22]. In patients infected with $M$. $t b$, droplets can be expelled from the bronchus and infect other persons [23]. The ability of these organisms to proliferate intracellularly and extracellularly is a prerequisite for them to cause active TB. Although majority of TB cases are cases of pulmonary TB, $M$. $t b$ can disseminate to and infect most organs and tissues to cause a variety of TB diseases [22].

The different manifestations and outcomes of $M$. $t b$ infection reflect the balance between the bacillus and the host defence mechanisms [13]. Macrophages constitute a major host defence mechanism against $M . t b$ infection [22]. Our study suggests that RF enhances the killing ability of macrophages against $M$. $t b$. This may be due to an increase in macrophage function and our data seem to indicate that macrophages are in a eustress condition during RF [5].

$\mathrm{RF}$ enhances interferon-gamma (IFN-gamma) and tumour necrosis factor-alpha (TNF-alpha) secretion by macrophages, and it has been suggested that RF induces classically activated macrophages in healthy subjects [5]. Activated macrophages can migrate to sites of inflammation where they encounter pathogens [24] and kill or suppress pathogen multiplication [22,25]. Pathogen killing is accomplished by an increased production of reactive oxygen species (ROS) and induction of iNOS to produce nitric oxide (NO) [24].

After pathogenic bacteria are engulfed into phagosomes, these bacteria are subject to killing by a variety of mechanisms including phagosome-lysosome fusion, the generation of reactive oxygen intermediates (ROI), and the generation of reactive nitrogen intermediates (RNI), particularly NO [26]. The production of ROI 
and RNI by innate immune cells is considered to be an effective host defence mechanism against microbial pathogens [9].

RF significantly induces ROS and NO in macrophages [5]. The significance of this toxic NO in host defence against $M$. $t b$ has been well documented both in vitro and in vivo, particularly in murine models [27]. The molecular mechanism of action of NO is not fully understood, although this molecule has been shown to inhibit aerobic respiration in mitochondria as well as in microbes [22,28]. Both pathogenic and non-pathogenic mycobacteria are internalised into macrophage phagosomes. While the non-pathogenic organisms are invariably killed by macrophages, the pathogenic organisms generally survive and grow in macrophages. The bactericidal effects of RNI against all bacterial species were restricted to the early stages of infection [29].

In patients infected with $M . t b$, droplets can be expelled from the bronchus and infect other persons. If discharge into a vessel occurs, the occurrence of extrapulmonary TB is likely [23]. A fatal form of extrapulmonary TB is infection of the bloodstream by mycobacteria; this form of the disease is called disseminated or miliary TB. The bacilli can then spread throughout the body, leading to multiorgan involvement in disease $[23,30]$. Our study suggests that RF significantly reduces the ability of serum to kill $M$. $t b$ at the end of RF.

The complement cascade can be activated through any of the three complement pathways: the classical, mannan-binding lectin and alternative pathways. In high concentrations of fresh nonimmune human serum, $M$. $t b$ activates the alternative pathway of complement and binds the $\mathrm{C} 3$ protein, resulting in enhanced phagocytosis of the microorganisms via complement receptors on human alveolar macrophages. However, the alternative pathway of complement is relatively inactive in the lung compared to the classical pathway [31]. Other studies have shown that $M$. $t b$ activates the classical pathway in addition to the alternative pathway in human serum [29].

A study by Scott et.al. demonstrated for the first time that in serum, C3 binding to $M$. $t b$ is rapid and is initiated by activation of the classical as well as the alternative pathway [31]. All of the complement cascades converge at the cleavage of $\mathrm{C} 3$ and the generation of the active fragments $\mathrm{C} 3 \mathrm{a}$ and $\mathrm{C} 3 \mathrm{~b}$. Opsonsation of foreign surfaces by covalent attachment of $\mathrm{C} 3 \mathrm{~b}$ induces three major functions: cell clearance by phagocytosis; amplification of complement activation by the formation of a surface-bound $\mathrm{C} 3$ convertase; and assembly of the C5 convertases. Cleavage of C5 induces the formation of a multiprotein pore complex (the membrane-attack complex (MAC)) [12]. The MAC is an ancient component of the immune defence system that results in lytic pores in pathogen membranes [32] that lead to cell lysis [12].

Our study shows that RF does not significantly affect the C3, iNOS or SOD levels in serum. These data are similar to the data from a study by Maliji et.al. that showed that fasting had no effect on C3 [33]. Although complement has a major role in the effect of serum against microorganisms, this role is less important in response to $M$. $t b$ [16]. The oxygen radical scavengers superoxide dismutase (SOD), catalase, mannitol, and diazabicyclooctane also do not have an effect on antimycobacterial activity [34]. ROI have little role in the defence against mycobacteria [26,34].

PBMC can produce NO in response to stimulation with tubercle bacilli. Studies have demonstrated no difference in NO production between healthy volunteers and patients with tuberculosis [35]. Our study revealed that RF enhances the ability of PBMC to kill $M$. $t b$ in early RF and reduces the ability of PBMC to kill $M$. $t b$ at the end of RF. This increase in antimicrobial activity may be caused by an increase in NO production by PBMC during RF, although no significant positive correlation between NO production and killing activity was observed $(\mathrm{r}=0.21 ; \mathrm{p}=0.47)$

No individual killing mechanism or cell type is sufficient to kill mycobacteria in vivo [9]. Our study reveals that killing of $M$. tb before RF is predominantly mediated by serum, the activity of which is significantly different $(p<0.05)$ than the activity of PBMC but not significantly different $(p>0.05)$ than the activity of macrophages. The activity of PBMCs to kill $M$. $t b$ on the $7^{\text {th }}$ day of Ramadan was significantly different $(\mathrm{p}<0.05)$ than the killing activity of serum and macrophages. The activity of macrophages to kill $M$. $t b$ on the $21^{\text {st }}$ day of Ramadan was significantly different $(\mathrm{p}<0.05)$ than the killing activity of PBMC and serum. This study suggests that serum defences to $M$. $t b$ during RF are weak, but this weakness is compensated by PBMC during early RF and by macrophages at the end of RF. A similar study by Latifynia et.al. has shown that despite a decline in the neutrophil phagocytic index and serum opsonisation index, the percentage of neutrophils participating in phagocytosis increased following fasting. Although there was a decrease in the opsonisation ability of the serum in this study, the increased percentage of opsonisation compensated for this defect [36].

$\mathrm{RF}$ enhances the beta-endorphin and endocannabinoid level of serum, PBMC and macrophages and induces different stress responses in serum, PBMC and macrophages [2,37,38]. Our laboratory data using correlation tests reveal that on $21^{\text {st }}$ day of RF, the killing ability of macrophages is significantly positively correlated $(\mathrm{r}=0.4 / \mathrm{p}=0.039)$ with beta-endorphin levels and significantly negatively correlation with endocannabinoid levels $(r=-0.384 / p=0.048)$ [2] and SOD levels in macrophages $(r=-0.444 / p=0.020)$ [5]. The 
other tested parameters were not significantly correlated. This study suggests that beta-endorphin and endocannabinoid, not $\mathrm{NO}$ as usually assumed, have predominant roles in the killing $M$. $t b$ during RF.

The effects of endorphin and endocannabinoids on the immune system indicate that beta-endorphin induces the differentiation of macrophages, and endocannabinoids are believed to control immune functions and play a role in immune homeostasis [39,40]. In addition, our study suggests that RF alters a cell type or mechanism in serum, PBMC or macrophages to induce killing of $M$. $t b$, but this signalling event or pathway remains unknown.

Activities during RF including fasting, praying and waking up at midnight for Suhoor are spirituality practices performed by Muslims in the lunar month of Ramadan that induce stress responses in the body [2]. Emotional stress can influence TB disease and TB disease is particularly common among individuals with mood disorders. The link between depression and TB is stronger than the link between depression and other medical illnesses. While the overall prevalence of TB has declined in the general population, certain groups remain at high risk for TB including the homeless, those who are HIV seropositive, individuals with a history of alcohol or drug abuse, and immigrants from a country in which TB is endemic. Many recipients of psychiatric services possess one or more of these risk factors, and consequently TB may be overrepresented in this population [41]. Other studies have shown that there is an increased risk of TB infection in patients diagnosed with rheumatoid arthritis $[42,43]$. This is contrary to our study that suggests that RF decreases the risk of TB infection.

The duration of treatment for TB has been reduced from 9-18 months to only 6 months [44]. During RF, Muslims who follow this tradition will abstain from ingesting any food or drink from sunrise to sunset each day for 30 days, and this includes abstaining from taking oral medications. Muslim TB patients who would like to fast even though their TB symptoms are predicted to become worse due to this practice, are offered directly observed therapy (DOT) before sunrise or after sunset. Likewise, LTBI/MDR-TB patients who are fasting can be advised to take their medications before sunrise or after sunset.

\section{Conclusions}

Our study suggests that RF reduces the ability of serum to kill $M$. $t b$ and enhances the ability of PBMC and macrophages to kill $M$. $t b$. The serum defence against $M$. $t b$ during RF is weak, but PBMC and macrophages can compensate for this weakness. This study also reveals that RF has beneficial effects and reduces the risk of TB infection in healthy subjects, but the effect of RF on LTBI or MDR-TB patients remains unknown.

\section{Acknowledgments}

The authors would like to thank Bio-Medic Brawijaya University for technical assistance.

\section{References}

[1]. Ibrahim WH, Habib HM, Jarrar AH and Al Baz SA (2008) Effect of Ramadan fasting on markers of oxidative stress and serum biochemical markers of cellular damage in healthy subjects. Ann Nutr Metab 53:175-81.

[2]. Lahdimawan A, Handono K, Indra MR and Prawiro SR (2013) Effect of Ramadan fasting on endorphin and endocannabinoid level in serum, PBMC and macrophage. IJPSI 2(3): 46-54

[3]. Lerner BH (1996) Can stress cause disease? Revisiting the tuberculosis research of Thomas Holmes, 1949-1961. Ann Intern Med 124(7):673-80.

[4]. Jonsdottir IH, Hoffmann P and Thorèn P (1997) Physical exercise, endogenous opioids and immune function. Acta Physiol Scand Suppl 640:47-50.

[5]. Lahdimawan A, Handono K, Indra MR and Prawiro SR (2013) Effect of ramadan fasting on classically activated, oxidative stress and inflammation of macrophage. IOSR Journal of Pharmacy 3(4):14-22.

[6]. Teixeira HC, Abramo C and Munk ME (2007) Immunological diagnosis of tuberculosis: problems and strategies for success. J Bras Pneumol 33(3):1806-3713.

[7]. Raviglione MC (2003) The TB epidemic from 1992 to 2002. Tuberculosis (Edinb) 83(1-3):4-14

[8]. Davies PD (2003) The world-wide increase in tuberculosis: how demographic changes, HIV infection and increasing numbers in poverty are increasing tuberculosis. Ann Med 35(4):235-43.

[9]. Chan ED, Chan J and Schluger NW (2001) What is the role of nitric oxide in murine and human host defense against tuberculosis? current knowledge. Am J Respir Cell Mol Biol 25(5):606-12.

[10]. North RJ and Jung YJ (2004) Immunity to tuberculosis. Annu Rev Immunol 22:599-623.

[11]. Chan ED, Morris KR, Belisle JT, Hill P, Remigio LK, Brennan PJ and Riches David WH (2001) Induction of inducible nitric oxide synthase-NO by lipoarabinomannan of Mycobacterium tuberculosis is mediated by MEK1-ERK, MKK7-JNK, and NF-KB signaling pathways. Infect Immun 69(4):2001-2010.

[12]. Lambris JD, Ricklin D and Geisbrecht BV (2008) Complement evasion by human pathogens. Nat Rev Microbiol 6(2):132.

[13]. Volpe E, Cappelli G, Grassi M, Martino A, Serafino A, Colizzi V, Sanarico N and Mariani F (2006) Gene expression profiling of human macrophages at late time of infection with Mycobacterium tuberculosis. Immunology 118(4):449-60.

[14]. Bouwens M, Afman LA and Müller M (2007) Fasting induces changes in peripheral blood mononuclear cell gene expression profiles related to increases in fatty acid $\beta$-oxidation: functional role of peroxisome proliferator-activated receptor $\alpha$ in human peripheral blood mononuclear cells. Am J Clin Nutr 86(5):1515-23.

[15]. Schlesinger LS, Bellinger-Kawahara CG, Payne NR and Horwitz MA (1990) Phagocytosis of Mycobacterium tuberculosis is mediated by human monocyte complement receptors and complement component C3. J Immunol 144(7):2771-80.

[16]. Swartz RP, Naai D, Vogel CW and Yeager H (1988) Differences in uptake of mycobacteria by human monocytes: a role for complement. Infect. Immun 56:2223. 
[17]. Misra UK, Kalita J, Tripathi GM and Bhoi SK (2013) Is $\beta$ endorphin related to migraine headache and its relief? Cephalalgia 33(5):316-22

[18]. Blomkalns AL, Stoll LL, Shaheen W, Romig-Martin SA, Dickson EW, Weintraub NL and Denning GM (2011) Low level bacterial endotoxin activates two distinct signaling pathways in human peripheral blood mononuclear cells. J Inflam 8:4.

[19]. Boyum A (1968) Isolation of mononuclear cells and granulocytes from human blood. Isolation of monuclear cells by one centrifugation, and of granulocytes by combining centrifugation and sedimentation at $1 \mathrm{~g}$. Scand J Clin Lab Invest Suppl 97:77-89.

[20]. Ting LM, Kim AC, Cattamanchi A and Ernst JD (1999) Mycobacterium tuberculosis Inhibits IFN- $\gamma$ transcriptional responses without inhibiting activation of STAT1. J Immunol 163(7):3898-906.

[21]. Palma C, Iona E, Ebensen T, Guzman CA and Cassone A (2009) The Toll-like Receptor 2/6 Ligand MALP-2 reduces the viability of mycobacterium tuberculosis in murine macrophages. Open Microbiol J 3:47-52.

[22]. Lamichhane G (2011) Mycobacterium tuberculosis response to stress from reactive oxygen and nitrogen species. Front Microbiol 2:176.

[23]. Knechel NA (2004) Tuberculosis: pathophysiology, clinical features, and diagnosis. Am J Crit Care 13:368-370.

[24]. Parameswaran N and Patial S (2010) Tumor Necrosis Factor-alpha signaling in macrophages. Crit Rev Eukaryot Gene Expr 20(2):87-103.

[25]. Ehrt S, Schnappinger D, Bekiranov S, Drenkow J, Shi S, Gingeras TR, Gaasterland T, Schoolnik G and Nathan C (2001) Reprogramming of the macrophage transcriptome in response to interferon-gamma and Mycobacterium tuberculosis: signaling roles of nitric oxide synthase-2 and phagocyte oxidase. J Exp Med 194:1123-1140.

[26]. Schluger NW and Rom WN (1998) The host immune response to tuberculosis. Am J Respir Crit Care Med (157)-3-679-691.

[27]. Raja A (2004) Immunology of tuberculosis. Indian J Med Res 120:213-232.

[28]. Brown GC (2001) Regulation of mitochondrial respiration by nitric oxide inhibition of cytochrome C oxidase. Biochim Biophys Acta 1504:46-57.

[29]. Jordao L, Bleck CK, Mayorga L, Griffiths G and Anes E (2008) On the killing of mycobacteria by macrophages. Cell Microbiol 10(2):529-48

[30]. Wang JY, Hsueh PR, Wang SK, Jan IS, Lee LN, Liaw YS, Yang PC and Luh KT (2007) Disseminated Tuberculosis: a 10-year experience in a medical center. Medicine (Baltimore) 86(1):39-46.

[31]. Ferguson JS, Weis JJ, Martin JL and Schlesinger LS (2004) Complement protein C3 binding to mycobacterium tuberculosis is initiated by the classical pathway in human bronchoalveolar lavage fluid. Infect Immun 72(5):2564-2573.

[32]. Aleshin AE, Schraufstatter IU, Stec B, Bankston LA, Liddington RC and DiScipio RG. (2012) Structure of complement C6 suggests a mechanism for initiation and unidirectional, sequential assembly of membrane attack complex (MAC). J Biol Chem 287:10210-22.

[33]. Maliji GH, Dordi Q, Omran MS and Habibi T (2006) Effects of ramadan fasting on complement components activities C3, C4, AND CH50 of human serum. J Ilam University of Med Sci 14(1):50-54.

[34]. Chan J, Xing Y, Magliozzo RS and Bloom BR (1992) Killing of virulent Mycobacterium tuberculosis by reactive nitrogen intermediates produced by activated murine macrophages. J Exp Med 175: 1111-1122.

[35]. Kwon OJ (1997) The role of nitric oxide in the immune response of tuberculosis. J Korean Med Sci 12(6): $481-487$.

[36]. Latifynia A, Vojgani M, Gharagozlou MJ and Sharifian R (2009) Neutrophil function (innate immunity) during Ramadan. J Ayub Med Coll Abbottabad 21(4):111-5.

[37]. Sharifi F, Masoud A, Makan R, Ziaei M and Hedayati M (2007) Evaluation of serum levels of IL- $1 \alpha$, TGF- $\beta$, TNF- $\alpha$, IFN- $\alpha$, IFNY, cortisol and immunoglobulins in islamic ramadan fasting. Iranian J Endocrinol Metab 9(2 (SN 34)):201-204.

[38]. Komaki G, Tamai H, Sumioki H, Mori T, Kobayashi N, Mori K, Mori S and Nakagawa T. (1990) Plasma beta-endorphin during fasting in man. Horm Res 33:239-243.

[39]. Hagi K, Inaba K, Sakuta H and Muramatsu S (1995) Enhancement of murine bone marrow macrophage differentiation by betaendorphin. The American Society of Hematology 86(4):1316-1321.

[40]. Pandey R, Mousawy K, Nagarkatti M and Nagarkatti P (2009) Endocannabinoids and immune regulation. Pharmacol Res 60(2):8592.

[41]. Trenton AJ and Currier GW (2001) Treatment of comorbid tuberculosis and depression prim care companion. J Clin Psychiatry 3(6):236-243.

[42]. Ehlers S. (2003) Role of tumour necrosis factor (TNF) in host defence against tuberculosis: implications for immunotherapies targeting TNF. Ann Rheum Dis 62(2):37-42.

[43]. Carmona L, Hernández-García C, Vadillo C, Pato E, Balsa A, González-Alvaro I, Belmonte MA, Tena X and Sanmartí R (2003) EMECAR study group. increased risk of tuberculosis in patients with rheumatoid arthritis. J Rheumatol 30(7):1436-9.

[44]. Iseman (2002) Tuberculosis therapy: past, present and future. ERJ 20(36):87s-94s 